
\title{
28 Research Square \\ Optogenetic control of mRNA localization and translation in live cells
}

Na Yeon Kim

Sangkyu Lee ( $\nabla$ sklee@ibs.re.kr)

Won Do Heo ( $\nabla$ wondo@kaist.ac.kr)

Method Article

Keywords: Optogenetics, Fluorescence microscopy, RNA imaging, RNA-protein interaction

Posted Date: March 26th, 2020

DOl: https://doi.org/10.21203/rs.3.pex-801/v1

License: (c) (1) This work is licensed under a Creative Commons Attribution 4.0 International License.

Read Full License 


\section{Abstract}

Numerous efforts have been made toward the goal of visualizing the spatiotemporal dynamics of single mRNA molecules, yet our capacity for precisely controlling their functions lags behind. Here, we present an optogenetic approach for manipulating the localization and translation of specific mRNAs in live cells. Our technique combines blue light-responsive protein-protein interactions with mRNA visualization modules to robustly and reversibly generate protein clusters that can trap specific mRNA molecules. This sequestration reduces the binding chance of mRNAs with ribosomes, thereby dramatically attenuating protein synthesis

\section{Introduction}

The localization and translation of mRNAs are regulated by complex and heterogeneous mechanisms in living systems. These mechanisms are believed to be tightly coupled to produce the finely tuned levels of protein synthesis required for cellular asymmetric structures and functions. To date, extensive effort has been invested in designing real-time visualization techniques to understand the dynamics of mRNA localization and translation at the single-molecule level. Although the many emerged techniques have revealed strong correlations between the spatiotemporal regulation of translation and specific cellular functions, their causalities have largely remained out of reach, due to a dearth of techniques for directly controlling specific mRNAs. Translation inhibitors and nucleotide-based strategies are used widely to manipulate mRNA localization and translation, but these techniques suffer from low spatial resolution and poor reversibility. Chemical compounds do not provide any specificity, and oligonucleotides typically require long lag times for their effects to manifest. Optogenetic approaches would be an ideal means to address these drawbacks. A variety of optogenetic methods exploiting photoreceptors from plants and bacteria have been developed to modulate a wide range of transcription and post-translational signaling, but little efforts have been made to control translation. Here, we present an optogenetic approach for robust and reversible inhibition of specific mRNAs in live cells.

\section{Reagents}

- DMEM (Gibco)

- Fetal bovine serum (Invitrogen)

$-0.25 \%$ Trypsin (Gibco)

- DPBS (Gibco)

- Lipofectamine ${ }^{\circledR}$ LTX (Invitrogen)

- Lipofectamine ${ }^{\circledR}$ RNAiMax (Invitrogen) 


\section{Equipment}

- T75 cell culture flask (Corning 430641U)

- $15 \mathrm{ml}$ tube (VWR 82050-276)

- 96-well plates ( $\mu$-Plate 96 Well ibiTreat; ibidi GmbH)

- Microporator (Neon ${ }^{\mathrm{TM}}$ Transfection System, Invitrogen)

- Nikon A1R confocal microscope (Nikon Instruments)

- Chamlide TC system (Live Cell Instruments)

\section{Procedure}

Cell culture

- HeLa (ATCC) was cultured in Dulbecco's modified Eagle's medium (DMEM; Gibco) supplemented with $10 \%$ FBS (Invitrogen) at $37^{\circ} \mathrm{C}$ with $10 \% \mathrm{CO} 2$.

- Prewarm culture media (DMEM $+10 \%$ FBS) and Trypsin in a $37^{\circ} \mathrm{C}$ water bath

- Aspirate culture media, gently rinse cells with DPBS, aspirate 1xPBS, then add $3 \mathrm{ml}$ Trypsin to the culture flask ( $75 \mathrm{~T}$ flask), and incubate under $37^{\circ} \mathrm{C}$ for 5 minutes to detach cells from the flask.

- Gently tap the flask to make sure all the cells are fully detached.

- Add $4 \mathrm{ml}$ of culture media to the culture flask, and collect all the cells.

- Transfer resuspended cells from the culture flask to a $15 \mathrm{ml}$ tube, then centrifuge resuspended cells in the $15 \mathrm{ml}$ tube at 2000rpm for 1 minutes. 
- Aspirate supernatant, and resuspended cell pellet with fresh culture media, pipette up and down gently, to make sure cells are fully resuspended.

- Count the number of cells using a hemocytometer.

Transfection

- Use the microporator to transfect all mRNA-LARIAT components to the cells (except for PAMmer). The condition for HeLa cell is two electroporation pulses of $980 \mathrm{~V}$ for $35 \mathrm{~ms}$. We suggest to determine the optimal amount of plasmid encoding LARIAT components and MS2 or RCas9 components depends on the target mRNA abundance. We used 1:1 ratio.

-Plate the transfected cells to 96 -well plate in $37^{\circ} \mathrm{C}$ with $10 \% \mathrm{CO} 2$ incubator overnight.

- On the next day, if RCas9 is used, transfect PAMmer using Lipofectamine ${ }^{\circledR}$ RNAiMax at least 6 hours before imaging.

Imaging

- Move the imaging plate to the microscope in the prewarmed environmental chamber.

- Set up software to acquire fluorescence images.

- Photostimuatlion can be performed in single 1s loops with a 488-nm laser at a light power density of $490 \mu \mathrm{W} \mathrm{mm}-2$ to generate a cluster.

\section{Troubleshooting}


If cluster is not formed after a single pulse of $488-\mathrm{nm}$ laser,

- Increase either laser power or exposure time.

If cluster is formed before any stimulation with 488-nm,

- change the fluorescent protein tagged with Cryptochrome 2. Depends on the fluorescent protein characteristics, Cryptochrome2 may aggregate.

- make sure to keep the sample dark before exposure to 488-nm.

If cluster do forms but decrease in translation of a target transcript is not observed,

- Depend on the target transcript half-life, the light stimulation time may vary.

- Change the ratio of LARIAT and mRNA visualization components when transfect (Ideally increase amount of LARIAT components).

\section{Time Taken}

$\sim$ two days.

\section{Anticipated Results}

Cluster will be observed as shown in Figure 1.

\section{References}

Lee, S. et al. Reversible protein inactivation by optogenetic trapping in cells. Nat Methods 11, 633-636 (2014).

Park, H. et al. Optogenetic protein clustering through fluorescent protein tagging and extension of CRY2. Nat Commun 8, 30 (2017).

\section{Acknowledgements}


We thank all members of the Heo laboratory for their support and advice. This work was supported by the Institute for Basic Science (no. IBS-R001-G1), KAIST Institute for the BioCentury, and KBRI basic research program through Korea Brain Research Institute funded by Ministry of Science and ICT (no. 19-BR-03-02), Republic of Korea.

\section{Figures}

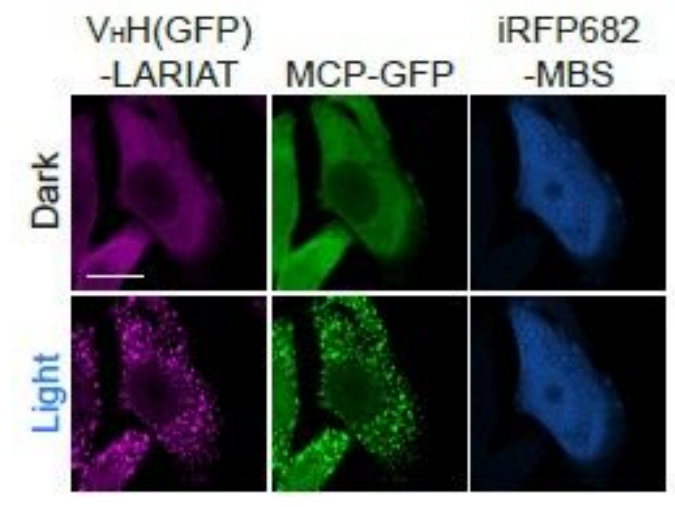

\section{Figure 1}

Fluorescence images of a HeLa cell co-expressing VHH(GFP)-LARIAT(mCherry-CRY2-VHH(GFP) and CIB1MP), MCP-GFP, and iRFP682-MBS illuminated with blue light (488 nm) at 10-sec intervals for 5 min.

\section{Supplementary Files}

This is a list of supplementary files associated with this preprint. Click to download.

- Video1.docx

- Video1.avi 